\title{
COLOUR SEGMENTATION FOR THE ANALYSIS OF PIGMENTED SKIN LESIONS
}

\author{
Ph. Schmid and S. Fischer
}

Signal Processing Laboratory, Swiss Federal Institute of Technology, 1015 Lausanne, Switzerland

\begin{abstract}
In this paper a colour segmentation scheme based on two-dimensional histogram analysis and the fuzzy c-means clustering technique is presented. The method is applied to digitised epiluminescence microscopy images of pigmented skin lesions and is used for the extraction of all homogeneously coloured regions. Methods to reduce the influence of small structures, hairs, bubbles, light reflexion, and other artefacts are proposed as pre and post-processing steps.
\end{abstract}

\section{INTRODUCTION}

One of the first steps for the computer aided analysis of pigmented skin lesions is the segmentation of the images based on colour or texture information. Texture analysis is important for the study of pigmented skin lesions, since the possible presence of relevant textures must be detected for diagnosis. These are important features for the diagnosis of malignant melanoma and other types of skin cancer (1). The texture detection should be preceded by a colour segmentation or a contour detection to extract the lesion border. Since colour also conveys significant information, a segmentation scheme based on the colour information seems to be suited as the first step. This allows for the isolation of the lesion from the healthy skin and for the extraction of homogeneously coloured regions. Further processing for the extraction of texture information may be carried out separately for each region (2).

Several segmentation schemes applied to images of skin lesions have been proposed, most of them dealing with the segmentation of clinical images. A method for extracting features from digitised images of pigmented skin lesions is presented in (3). This method is based on a spherical transformation of the $R G B$ colour space and on the Karhunen-Loève transform. Six different segmentation methods for the extraction of the lesion boundary are compared in (4). Other methods for the extraction of skin tumour boundaries are presented in $(5,6,7)$.

The segmentation scheme we propose in this paper is an extension of a method proposed in (8) and is described in the next sections.

\section{SEGMENTATION SCHEME}

The colour segmentation scheme proposed in (8) consist of two-steps: histogram information of the three $R G B$ colour components is used to derive the number of clusters and the approximate location of their centre. A coarse segmentation is obtained by labelling all pixels located in a close neighbourhood of each cluster centre. The remaining pixels are labelled using the fuzzy c-means (FCM) technique. This segmentation method has the following limitations:

- The three histograms, one for each colour component, are analysed separately. Since the colour components are usually strongly correlated the use of a multidimensional histogram would improve the detection of colour clusters.

- The authors propose to coarsely segment the image using the cluster centres computed from the histograms and to use the fuzzy c-means technique for the fine segmentation, although this technique recomputes the cluster centres after each recursion and from all the pixels.

The latter limitation can be overcome if the cluster centres are not recomputed and thus considered as sufficiently well approximated. In that case a clustering based on nearest neighbour criteria should be used instead (9). We propose an extension of the method proposed in (8) using two-dimensional histogram analysis. The full method is described in the following sections. The fuzzy c-means technique is described in section 2.4 .

\subsection{Material}

The processed images are digitised epiluminescence microscopy (ELM) slides of pigmented skin lesions $(10,1)$. This technique makes use of an oil immersion to render the skin translucent. The resulting images show structures located at lower skin layers. The number of possible colours, the different textures, and the imprecise border location makes the analysis of such images difficult.

\section{$2.2 \quad$ Filtering}

Filtering is an important pre-processing step for the image segmentation when based on colour information. Small structures should be removed in order 
to reduce the over-segmentation resulting from the colour space clustering. In the following sections three efficient methods among the existing ones are described.

2.2.1 Low-Pass Filtering. The simplest way to reduce the over segmentation is to smooth out the entire image by using a low-pass filter. It is suggested to use a separable filter with a Gaussian shape (11). The drawback of filtering the original image is that the lesion borders are smoothed out as well as the image structure. This makes the border location even more imprecise. Better result can be obtained using a median filter.

2.2.2 Median Filtering. We use a non-linear filter which assigns to each pixel the median pixel-value over a neighbourhood of a given size. This filter reduces the influence of small structures, like hair, on the segmentation result. The neighbourhood used for the median filter depends on the image resolution. In this work, a $9 \times 9$ neighbourhood has been used for images of size $450 \times 450$ pixels showing a complete lesion. Even if the lesions vary in size, this value can be kept fixed. The computation time is much larger for this filter than for a low-pass filter.

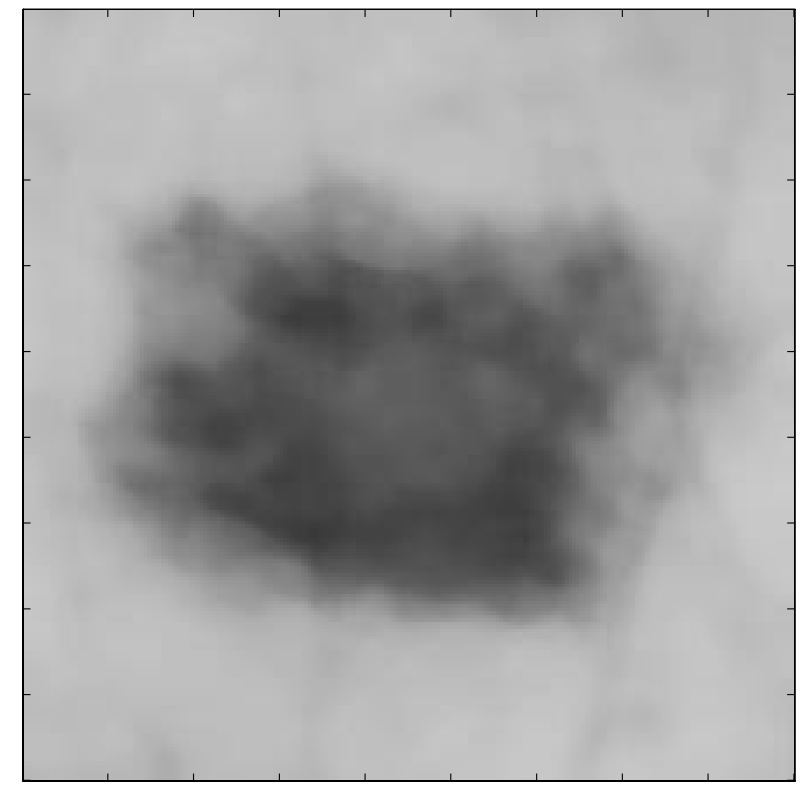

Figure 1: Result after median filtering applied to the image shown in Fig. 3. Inner structures and hair have been smoothed out.

2.2.3 Grey Level Morphology. Hair can be removed using morphological closing (hair is dark, skin is light). The drawback is that this operation removes small texture structures at the lesion boundary. These structures are part of the lesion and must not be removed. Thus the morphological filtering must begin with an opening operation with a structuring element diameter greater than the smallest texture element. This operation may be followed by a closing with a structuring element with smaller diameter, but larger than the hair width. The second morphological operation does not influence significantly the histogram analysis (see section 2.3), but reduces strongly the over-segmentation. Here we use a structuring element with cylindrical shape. For morphological concepts and operators description see (11).

Unfortunately the use of morphological tools to smooth the input image is too radical. The morphological filtering results in a sort of pre-segmentation of the image. This results in a large amount of sharp peaks in the histogram.

\subsection{Histogram Analysis}

In (8), the histogram of each colour component is used to determine the number of colour classes. As explained in section 2, this may hide some relevant maxima. We thus propose to use a two-dimensional histogram to extract information about the number of clusters and their location. Using a threedimensional histogram would be optimal, but in order to reduce the amount of data, the $R G B$ input is mapped onto a two-dimensional space, as explained later in section 2.6.3. The number of valid classes correspond to the number of dominant peaks in the histogram. This information may be extracted only after the histogram has been smoothed in order to suppress small local maxima. We have used the morphological filtering operation (closing-opening) to smooth the histogram. The ideal value, or range of values, for the structuring element diameter have been determined empirically. We suggest the use of a cylindrical shape for the structuring element which allows to control the minimum distance between two neighbouring peaks.

\subsection{Clustering}

The fuzzy c-means technique has proved to be a robust clustering technique when applied to our problem. Given the number of clusters, the two recurrent equations given below are used (8):

$$
\begin{aligned}
U_{i k} & =\left(1+\sum_{\substack{j=1 \\
j \neq i}}^{c}\left(\frac{\left\|\mathbf{x}_{k}-\mathbf{v}_{i}\right\|}{\left\|\mathbf{x}_{k}-\mathbf{v}_{j}\right\|}\right)^{2 /(m-1)}\right)^{-1} \\
v_{i} & =\frac{\sum_{k=1}^{n}\left(U_{i k}\right)^{m} \cdot \mathbf{x}_{k}}{\sum_{k=1}^{n}\left(U_{i k}\right)^{m}}
\end{aligned}
$$

where $\mathbf{x}_{k}$ is the $k^{t h}$ sample vector, $n$ the number of sample vectors, $c$ the number of classes, $\mathbf{v}_{i}$ the $i^{t h}$ class centre, $U_{i k}$ the fuzzy membership of $\mathbf{x}_{k}$ to class $i$, and $m$ is the weighting exponent. $\|\cdot\|$ is any inner product norm of the form $\|\mathbf{v}\|=\mathbf{v}^{T} \mathbf{A} v, \mathbf{A}$ being a positive definite matrix. The weighting ex- 


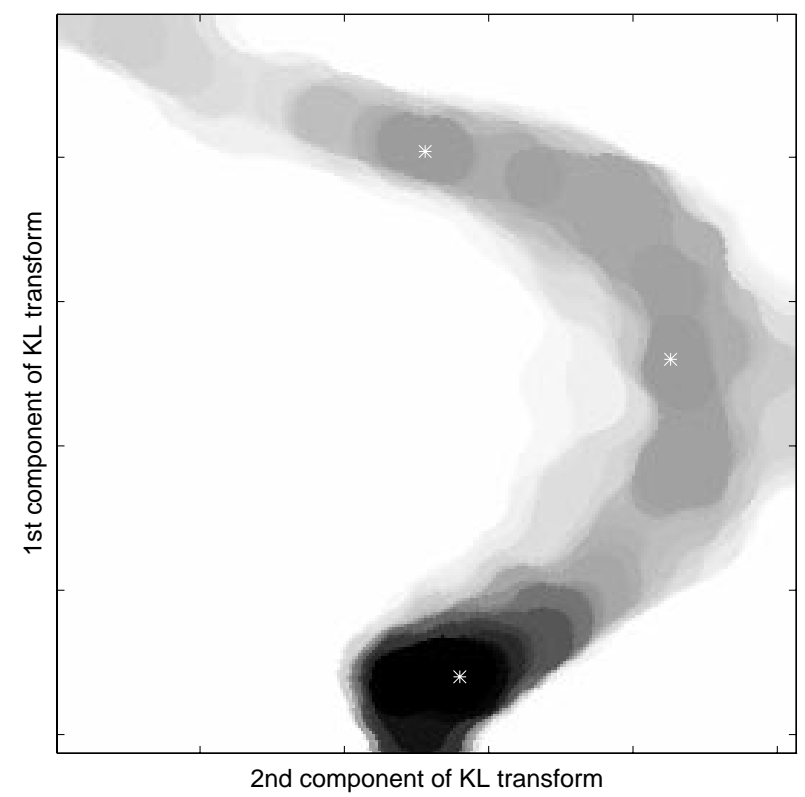

Figure 2: Two dimensional histogram corresponding to the images shown in Fig. 3 and 4. Smoothing has been computed using morphological closing operation with structuring element of cylindrical shape and diameter 21 pixels. Morphological opening has also been applied to perform filtering, but with smaller diameter (10 pixels) to avoid suppression of narrow peaks. The three valid maxima are marked in white.

ponent $m$ defines the fuzziness of the membership values. When $m$ equals infinity, the fuzzy membership becomes equal to $1 / c$ for all classes, except if the sample vector $\mathbf{x}_{k}$ corresponds to a cluster centre. In that case, the fuzzy membership is equal to 1. Therefore, large values of $m$ should be avoided, since this increases the fuzziness of the system.

The fuzzy membership of a sample vector in a colour class depends on its distance to this class and to the other classes. Sample vectors located at a given distance from an isolated cluster centre will have a larger fuzzy membership than vectors located at the same distance from another cluster centre, but surrounded by other clusters.

\subsection{Region cleaning}

The segmented image is usually over segmented. That is, small structures like hair, light reflections, or pigmented structures may influence the segmentation and thus the number of regions. We have underlined the need of filtering the original image before segmentation(section 2.2). To smooth the borders and to suppress small regions, morphological operators like closing-opening and extraction of connected components have been used (11).

\subsection{Colour Spaces}

Colour space change may improve the ability of a segmentation scheme to discriminate different colour components. Depending on the colour space transformation chosen, the obtained components may be mainly sensitive to some chromatic wavelengths. This may be useful if the possible colours to be detected are known a priory. Different colour spaces have been evaluated and are described in the following subsections.

2.6.1 Intensity, Hue, and Saturation components. This colour space separates the $R G B$ input image into one luminance and two chrominance components, namely the hue and the saturation (11). We could experience that in general the luminance component contains most of the information about the texture and boundary location. The hue component is usually homogeneous (with small variance), except when the lesion contains bluish or reddish regions. This is due the dominance of brown colour which results from the skin pigmentation. The saturation component allows to differentiate between different shades of the same colour, for example light and dark brown. Thus, if using this colour space with the proposed segmentation scheme, one should consider the intensity and saturation components.

2.6.2 Uniform Colour Spaces. These colour spaces attempt to introduce the notion of colour distance (Euclidean distance) (12). They are adapted to the human visual system (HVS) and may allow for a better separation between the colours. That is, two different colours should be distant whereas two shades of the same colour should be close. When using these colour spaces, one should consider the luminance component and the principal component of the Karhunen-Loève transform (see section 2.6.3) of the two chrominance components.

2.6.3 Karhunen-Loève Transform. The most appropriate colour space transformation when using the proposed segmentation scheme is, as far as we could experience, the Karhunen-Loève (KL) transform. A new colour base is computed according to the direction of maximum variance. The KarhunenLoève transform, also called Hotelling transform or principal components analysis (11), is the projection of the three components on the eigenvectors of their covariance matrix, computed using the following equations (11):

$$
\begin{aligned}
\mathbf{m}_{x} & =\frac{1}{M} \cdot \sum_{k=1}^{M} \mathbf{x}_{k}, \\
\mathbf{C}_{x} & =\frac{1}{M} \cdot \sum_{k=1}^{M} \mathbf{x}_{k} \cdot \mathbf{x}_{k}^{T}-\mathbf{m}_{x} \cdot \mathbf{m}_{x}^{T},
\end{aligned}
$$




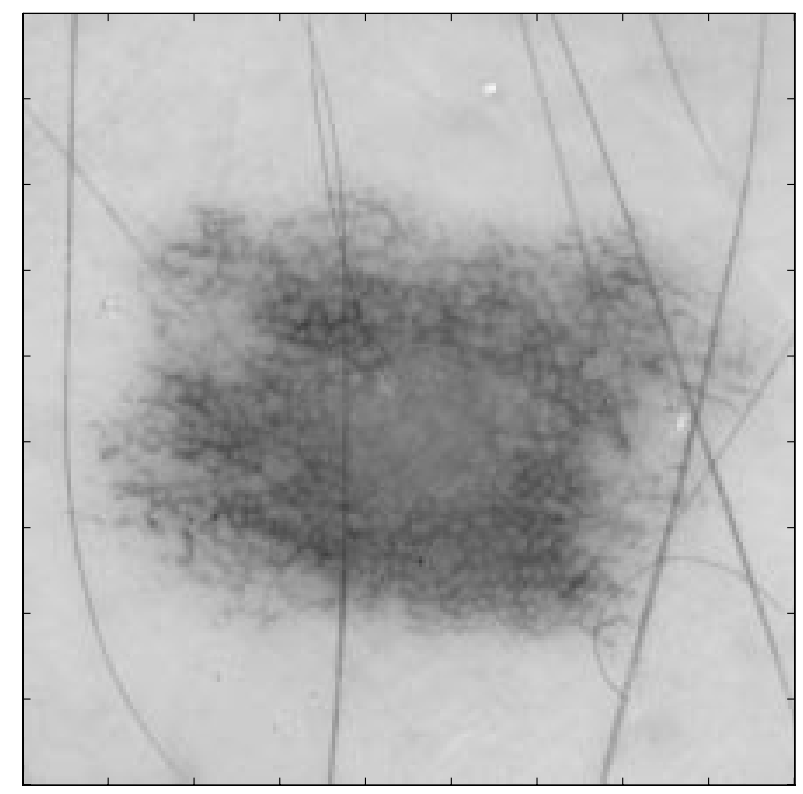

Figure 3: Principal component of KL transform of original $R G B$ image. This component may be identified to the luminance or intensity component that one can get in other colour spaces. Most of the texture information is mapped onto that component. Note: this image has been scaled and re-quantised using a gamma correction to enhance the visual quality.

where $M$ is the number of $R G B$ colour vectors $\mathbf{x}_{k}$ (or pixels in this case) and $\mathbf{m}_{x}$ is the mean sample vector. Thus, defining a matrix $\mathbf{A}$ whose lines are composed of the eigenvectors placed in increasing order, the Karhunen-Loève transform is defined by (11):

$$
\mathbf{y}=\mathbf{A} \cdot\left(\mathbf{x}-\mathbf{m}_{x}\right)
$$

Figures 3 and 4 show the two principal components obtained with the KL transform in decreasing order (of variance). The third component is not shown because of its small variance. The two principal components contain most of the structure information and may be used as a two dimensional approximation of the $R G B$ input image. Thus, combining this transform and the proposed segmentation scheme leads to the best results.

\section{RESULTS}

Applications of our segmentation scheme are shown in Fig. 5 and 6 . The former shows a segmentation result without any pre or post-processing, whereas the latter shows a result using the complete scheme. The effect of the pre and post-processing operations is clearly visible. The hair and the textured regions located on the lesion border do not influence the border location. The smoothness of the border is controlled by the post-processing operation.

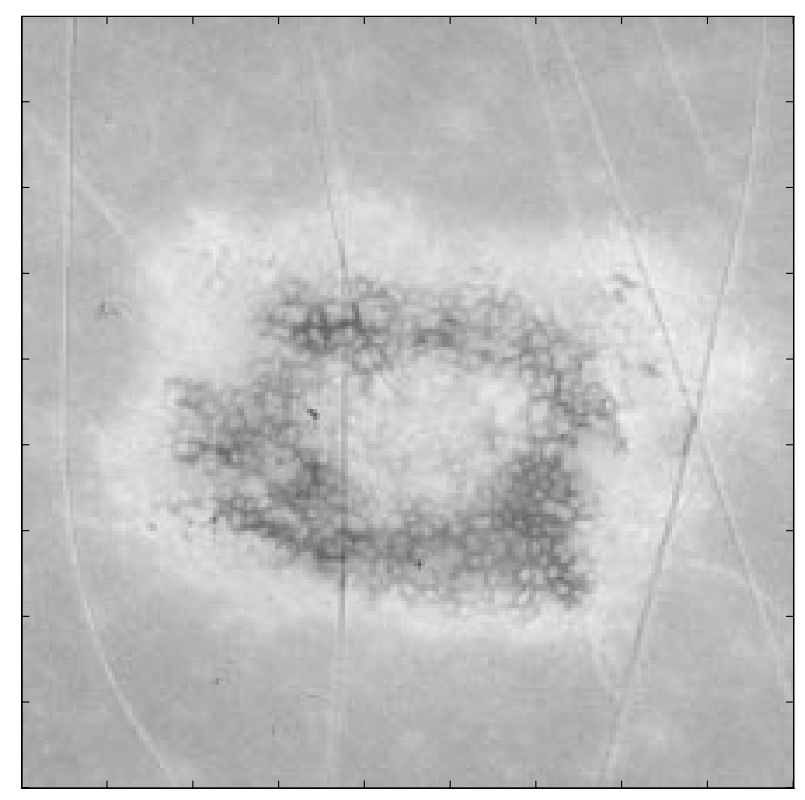

Figure 4: Second component of KL transform of original $R G B$ image. This component is already less detailed than the principal component. The boundary between homogeneous regions is still visible. Note: this image has been scaled and re-quantised using a gamma correction to enhance the visual quality.

\section{DISCUSSION}

The main problem is the presence of a high and sharp peak in almost all two-dimensional histograms we could compute from images of pigmented skin lesions. This peak corresponds to the healthy skin that surrounds the lesion and which has a small colour variance when compared to the lesion. Indeed, regions within the lesion usually have a large variance in the colour distribution. The presence of such a peak renders the histogram analysis even more sensitive to the degree of smoothing. This peak problem depends strongly on the image size occupied by the lesion. Thus, an alternative to our segmentation scheme would be to determine first the lesion border and then to segment the lesion using our method. Methods using active contours may give good results and are being investigated.

We could experience that the values used for the structuring element diameter (see section 2.3) can be determined empirically and can be used for most of the images. In some cases however, these values must be tuned to get the right number of colour classes. Therefore, the segmentation method we have proposed in this paper is to be considered as being supervised. However, this is not a real drawback since analysis of pigmented skin lesions for diagnosis purposes is supervised by the physician. 


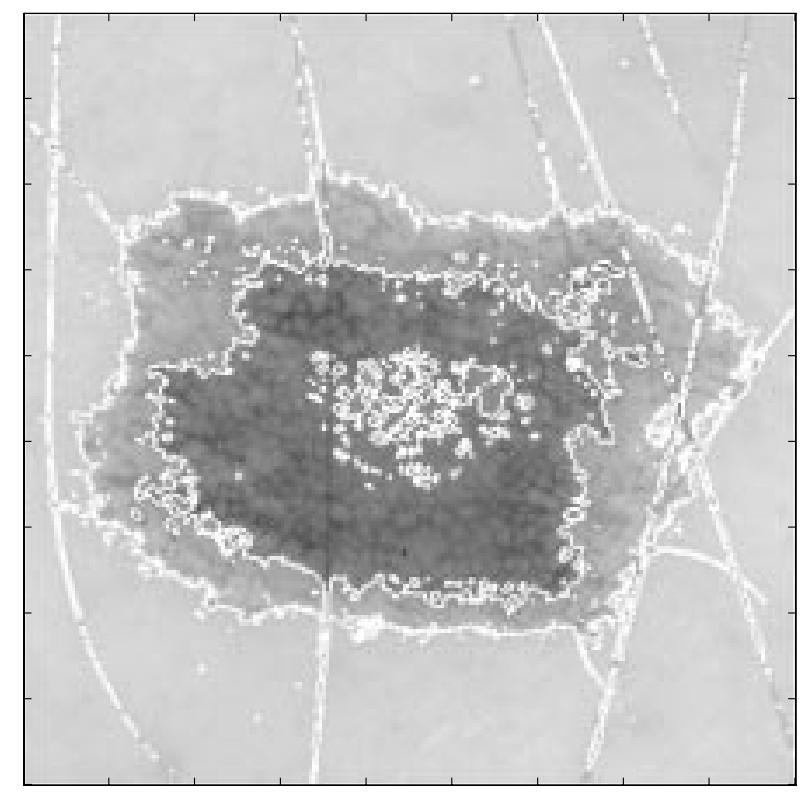

Figure 5: Image of Fig. 3 segmented using histogram information shown in Fig. 2.3 and the fuzzy c-means clustering technique. There is no pre or post-processing. Pigment structure and hair influence strongly the border location.

\section{REFERENCES}

1. W. Stolz, O. Braun-Falco, M. Landthaler P. Bilek, and A. B. Cognetta. Color Atlas of Dermatoscopy. Blackwell Science, London, UK, 1994.

2. S. Fischer, Ph. Schmid, and J. Guillod. "Analysis of skin lesions with pigmented networks". In Int. Conf. Image Process., Vol. 1, pp. 323-326, Lausanne, 16-19 September 1996. IEEE Signal Processing Society.

3. S. E. Umbaugh, R. H. Moss, W. V. Stoecker, and G. A. Hance. "Automatic color segmentation algorithms with application to skin tumor feature identification". IEEE Engineering in Medicine and Biology, Vol. 12, No. 3, pp. 7581, September 1993.

4. G. A. Hance, S. E. Umbaugh, R. H. Moss, and W. V. Stoecker. "Unsupervised color image segmentation; with application to skin tumor borders". IEEE Engineering in Medicine and Biology, Vol. 15, No. 1, pp. 104-111, January/February 1996.

5. J. E. Golston, R. H. Moss, W. V., and Stoecker. "Boundary detection in skin tumor images: An overall approach and a radial search algorithm". Pattern Recognition, Vol. 23, No. 11, pp. 12351247, 1990.

6. F. Ercal, M. Moganti, W. V. Stoecker, and R. H. Moss. "Detection of skin tumor boundaries in color images". IEEE Transactions on Medical Imaging, Vol. 12, No. 3, pp. 624-627, September

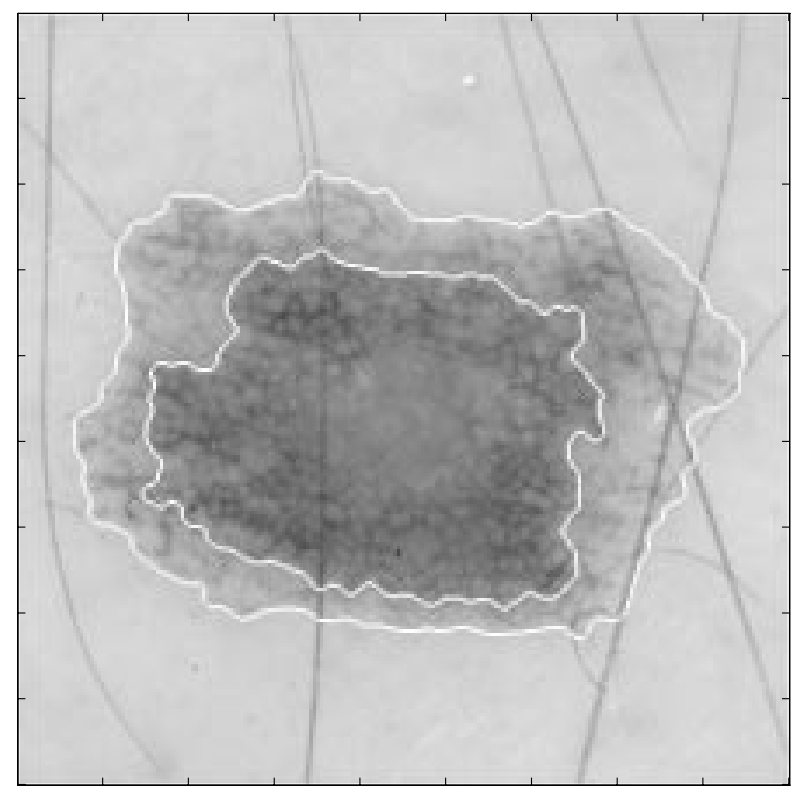

Figure 6: Image of Fig. 3 segmented using histogram information shown in Fig. 2.3 and the fuzzy c-means clustering technique. The original image has been filtered using a median filter (section 1) before processing. Small regions have been extracted and region border smoothed using morphological tools (section 2.5). Pigment structure and hair has no influence on the segmentation result.

1993.

7. W.E. Denton, A. W. G. Duller, and P. J. Fish. "Boundary detection for skin lesion: an edge focusing algorithm". In Image Processing and its Applications, No. 410 in Conference Publication, pp. 399-402. IEEE, 4-6 July 1995.

8. Y. W. Lim and S. U. Lee. "On the color image segmentation algorithm based on the thresholding and the fuzzy c-means techniques". Pattern Recognition, Vol. 23, No. 9, pp. 935-952, 1990.

9. R. Schalkoff. Pattern recognition: statistical, structural and neural approaches. Wiley, 1992.

10. M. Binder, M. Schwarz, A. Winkler, A. Steiner, A. Kaider, P. Wolff, and H. Pehamberger. "Epiluminescence microscopy; a useful tool for the diagnosis of pigmented skin lesions for formally trained dermatologists". Arch. Dermatol., Vol. 131, pp. 286-291, March 1995.

11. R. C. Gonzalez and P. A. Wintz. Digital Image Processing. Addison-Wesley, Reading, MA, 1992.

12. G. Wyszecki and W. S. Stiles. Color science: concepts and methods, quantitative data and formulae. Wiley, New-York, 1982. 